\title{
A Large-Eddy Simulation Study of Thermal Effects on Turbulent Flow and Dispersion in and above a Street Canyon
}

\author{
SEUNG-BU PARK AND JONG-Jin BAIK \\ School of Earth and Environmental Sciences, Seoul National University, Seoul, South Korea \\ SIEGFRIED RAASCH AND MARCUS OLIVER LETZEL* \\ Institute of Meteorology and Climatology, Leibniz University of Hannover, Hannover, Germany
}

(Manuscript received 1 September 2011, in final form 23 November 2011)

\begin{abstract}
Thermal effects on turbulent flow and dispersion in and above an idealized street canyon with a street aspect ratio of 1 are numerically investigated using the parallelized large-eddy simulation model ("PALM"). Each of upwind building wall, street bottom, and downwind building wall is heated, and passive scalars are emitted from the street bottom. When compared with the neutral (no heating) case, the heating of the upwind building wall or street bottom strengthens a primary vortex in the street canyon and the heating of the downwind building wall induces a shrunken primary vortex and a winding flow between the vortex and the downwind building wall. Heating also induces higher turbulent kinetic energy and stronger turbulent fluxes at the rooftop height. In the neutral case, turbulent eddies generated by shear instability dominate mixing at the rooftop height and appear as band-shaped perturbations in the time-space plots of turbulent momentum and scalar fluxes. In all of the heating cases, buoyancy-generated turbulent eddies as well as shear-generated turbulent eddies contribute to turbulent momentum and scalar fluxes and band-shaped or lump-shaped perturbations appear at the rooftop height. A quadrant analysis shows that at the rooftop height, in the neutral case and in the case with upwind building-wall heating, sweep events are less frequent but contribute more to turbulent momentum flux than do ejection events. By contrast, in the case with street-bottom and downwind building-wall heating, the frequency of sweep events is similar to that of ejection events and the contribution of ejection events to turbulent momentum flux is comparable to that of sweep events.
\end{abstract}

\section{Introduction}

There has recently been growing interest in flow and air quality in built-up urban areas as urbanization continues. Sufficient understanding of the basic fluid dynamics of building-scale or street canyon-scale flow and dispersion to allow better simulation and prediction of flow and air quality in built-up urban areas is lacking-fundamentally because of the turbulent nature of building-scale or street canyon-scale flow and

* Current affiliation: Ingenieurbüro Lohmeyer GmbH \& Co. KG, Karlsruhe, Germany.

Corresponding author address: Jong-Jin Baik, School of Earth and Environmental Sciences, Seoul National University, Seoul, South Korea.

E-mail: jjbaik@snu.ac.kr dispersion. There are many factors that affect buildingscale or street canyon-scale flow and dispersion, including the shape and arrangement of buildings, ambient wind speed and direction, building-surface or street-bottom heating by solar radiation, and turbulence induced by vehicles. Among the above factors, heating of a building surface or street bottom is the main source of thermal turbulence.

Thermal effects on mean flow in street canyons have been investigated through wind-tunnel experiments, field observations, and numerical simulations (Sini et al. 1996; Kim and Baik 1999; Uehara et al. 2000; Louka et al. 2002; Kovar-Panskus et al. 2002; Xie et al. 2006; Offerle et al. 2007). These studies indicate that changes in the thermal environment (e.g., heating position and heating intensity) alter mean flow in street canyons. For example, a primary vortex in an isothermal street canyon with the ambient wind perpendicular to the 
street axis can strengthen or weaken and more vortices can be induced when the building surface or street bottom is heated.

Although heating in street canyons also affects turbulence therein, less attention has been paid to thermal effects on turbulence. There are some studies of turbulence structure in street canyons (Rotach 1993, 1995; Cui et al. 2004; Christen et al. 2007). Rotach (1993) analyzed organized turbulence structure using measurement data and emphasized the importance of sweep events for the exchange of canyon air and air above the rooftop height. Rotach (1995) investigated turbulence statistics in and above a street canyon over a long period. The turbulence statistics are shown to be strongly stability dependent. Cui et al. (2004) simulated turbulent flow in an isothermal street canyon of a unity street aspect ratio and analyzed turbulent events at the rooftop height by performing a quadrant analysis. They showed that weak ejection events are dominant in frequency of occurrence, but fewer strong sweep events contribute to most of the total momentum transport at the rooftop height. By analyzing measurement data, Christen et al. (2007) also emphasized the dominance of sweep events in the turbulent momentum and sensible heat fluxes in large parts of a street canyon and close to the roofs. Several studies indicated that turbulence structures in street canyons are influenced by large-scale turbulent motions (e.g., low-speed streaks and streamwise vortices) appearing above building canopies (Kanda et al. 2004; Coceal et al. 2007; Inagaki and Kanda 2010).

When the building surface or street bottom is heated, the turbulence structure can change significantly because of turbulent eddies generated by buoyancy as well as turbulent eddies generated by shear instability. There are few studies that focus on turbulence structure in a street canyon in which the building surface or street bottom is heated. A turbulence study of plant canopies by Shen and Leclerc (1997) showed that the contribution of ejection events to turbulent momentum transport becomes large as the heating intensity just above and below the treetop height increases. It would be interesting to examine turbulence structure in a nonisothermal street canyon. This study attempts to investigate thermal effects on turbulence structure as well as mean flow in and above a street canyon and to clarify the roles of shear-generated and buoyancy-generated turbulent eddies in momentum and scalar transport. For this, a large-eddy simulation (LES) model is used.

The LES model and simulation setup are described in section 2. In section 3, the LES model is validated using wind-tunnel experimental data. The results are presented and discussed in section 4 . A summary and conclusions are given in section 5.

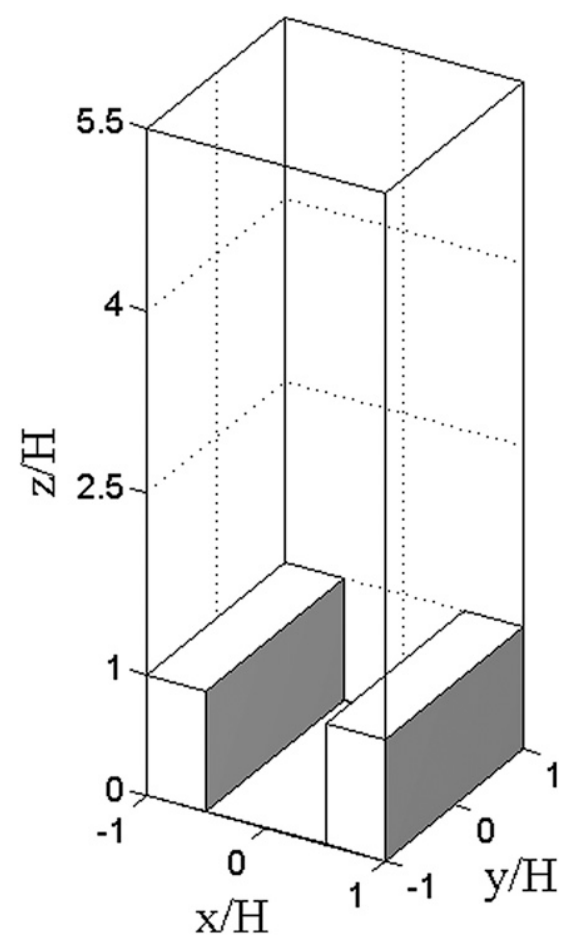

FIG. 1. An illustration of the computational domain and building configuration. Here, $H$ is the building height.

\section{Model description and simulation setup}

The numerical model used in this study is the parallelized LES model developed at the Leibniz University of Hannover (Raasch and Schröter 2001; Letzel et al. 2008) and known as "PALM." It is based on the filtered, Boussinesq equations. The governing equations are the momentum equation, thermodynamic energy equation, subgrid-scale (SGS) turbulent kinetic energy (TKE) equation, and passive scalar equation. The SGS turbulent fluxes are parameterized using the 1.5-order Deardorff (1980) scheme, which uses SGS TKE to calculate eddy viscosity. Further details of PALM are described in Letzel et al. (2008).

A simple urban canopy with two buildings (20-m height) and a street canyon (20-m width) is considered in this study (Fig. 1). The street aspect ratio is 1 . The size of the computational domain is $40 \mathrm{~m}$ in the $x$ direction (streamwise direction), $40 \mathrm{~m}$ in the $y$ direction (spanwise direction), and $109 \mathrm{~m}$ in the $z$ direction (vertical direction). The grid size in both the $x$ and $y$ directions is $0.5 \mathrm{~m}$. In the $z$ direction, the grid size is uniform up to $z=50 \mathrm{~m}$ and then gradually increases with an expansion ratio of 1.08. Considering that infinitely long street canyons are repeated in the streamwise direction, the cyclic boundary condition is applied at the horizontal boundaries for velocity components and SGS TKE. The 
TABLE 1. Heating position, streamwise wind speed at $z=2 H$, temperature difference between a heated surface and the air at $z=$ $2 \mathrm{H}$, and the bulk Richardson number in the $\mathrm{NH}, \mathrm{UH}, \mathrm{BH}$, and $\mathrm{DH}$ cases.

\begin{tabular}{llccc}
\hline \hline & & $U_{2 H}$ & $T_{w}-T_{2 H}$ & \\
Cases & \multicolumn{1}{c}{ Heating position } & $\left(\mathrm{m} \mathrm{s}^{-1}\right)$ & $(\mathrm{K})$ & $R_{b}$ \\
\hline NH & No heating & 2.6 & 0 & 0 \\
UH & Upwind building wall & 2.4 & 20 & -2.27 \\
BH & Street bottom & 2.3 & 20 & -2.47 \\
DH & Downwind building wall & 2.2 & 20 & -2.70 \\
\hline
\end{tabular}

zero-gradient boundary condition is applied at the top boundary. The initial ambient wind direction is perpendicular to the spanwise direction, and the initial vertical profile of the ambient wind speed is logarithmic. The initial volume flow rate at the inflow boundary is conserved to maintain quasi-steady mean flow in the model domain, and the Coriolis effect is not considered in this study.

At the grid points closest to all of the solid surfaces, Monin-Obukhov (MO) similarity is employed in the momentum equation and the passive scalar equation. In the previous version of PALM, heat fluxes that are based on MO similarity or prescribed constant heat fluxes are applied at the bottom and building-top surfaces, whereas only prescribed constant heat fluxes are applied at the building-wall surfaces. To take into account the heat exchange between the ambient air and the building-wall surfaces, a method that calculates heat fluxes at the building-wall surfaces on the basis of MO similarity is developed for this study. In general, the applicability of MO similarity (for heat fluxes at building scale) is not ensured. Nevertheless, MO similarity showed good performance in the validation tests in this study (section 3), and therefore it was selected as a temperature wall function.

Passive scalars are emitted at all grid points of the street bottom; the emitted passive scalar flux is $5 \mathrm{~kg} \mathrm{~m}^{-2} \mathrm{~s}^{-1}$. The passive scalar concentration is set equal to 0 and the temperature is fixed at $300 \mathrm{~K}$ at the inflow boundary to maintain quasi-steady mean states of scalar and temperature fields. The situation of zero passive scalar concentration at the inflow boundary corresponds to a situation in which ambient fresh air continuously flows into the domain. PALM is integrated for $3 \mathrm{~h}$, and the simulation data for the last $30 \mathrm{~min}$ are used for analysis.

To investigate thermal effects on turbulent flow and dispersion, four simulations are conducted with different heating positions: a no-heating $(\mathrm{NH})$ case (control case), a case with upwind building-wall heating (UH), a case with street-bottom heating $(\mathrm{BH})$, and a case with (a)

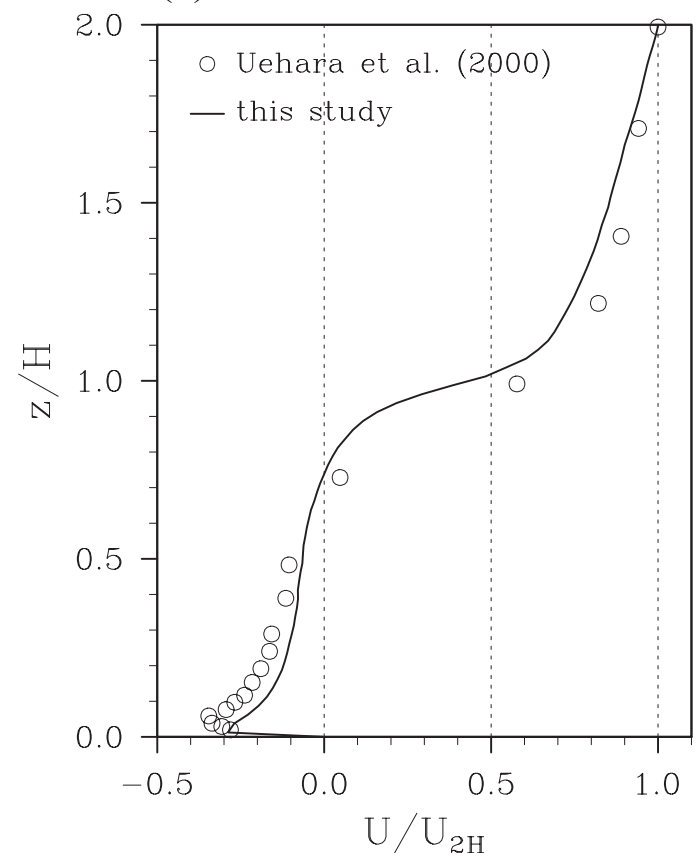

(b)

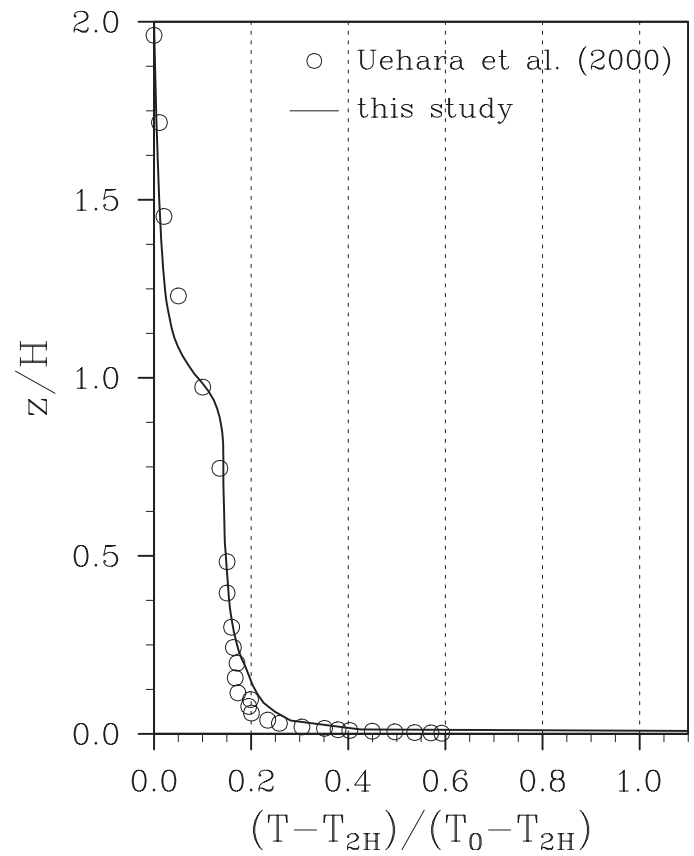

FIG. 2. Vertical profiles of (a) normalized streamwise horizontal velocity and (b) normalized temperature at the center of a street canyon in the current numerical simulation and in the wind-tunnel experiment of Uehara et al. (2000). Here, $U_{2 H}$ is the streamwise horizontal velocity at $z / H=2, T_{2 H}$ is the temperature at $z / H=2$, and $T_{0}$ is the temperature at the bottom surface. 
downwind building-wall heating $(\mathrm{DH})$. Table 1 shows the heating position, streamwise wind speed at $z=2 \mathrm{H}$ $(40 \mathrm{~m})$, temperature difference between a heated surface and the air at $z=2 \mathrm{H}$, and bulk Richardson number in the $\mathrm{NH}, \mathrm{UH}, \mathrm{BH}$, and $\mathrm{DH}$ cases. Here, the bulk Richardson number is defined as

$$
R_{b}=\left[\left(g / T_{2 H}\right)\left(T_{2 H}-T_{w}\right) / 2 H\right] /\left(U_{2 H} / 2 H\right)^{2},
$$

where $H$ denotes the building height and $T_{2 H}$ and $U_{2 H}$ denote the temperature and streamwise wind speed at $z=2 H$, respectively. Temperature $T_{w}$ is the temperature of the heated building wall or street bottom, and $g$ is the gravitational acceleration. Streamwise wind speeds at $z=2 H\left(2.2-2.6 \mathrm{~m} \mathrm{~s}^{-1}\right)$ are strong enough to induce a primary vortex and corner vortices in the street canyon, and the temperature of a heated surface $(320 \mathrm{~K})$ is high enough to affect flow therein.

\section{Validation}

The LES model is validated using velocity and scalar concentration data obtained from wind-tunnel experiments. First, the flow in a bottom-heated street canyon is validated against the wind-tunnel data of Uehara et al. (2000). Figure 2 shows the vertical profiles of normalized streamwise horizontal velocity and normalized temperature in the numerical simulation and in the windtunnel experiment. In the numerical simulation and windtunnel experiment, an array of cubic model buildings is considered. In the wind-tunnel experiment, the bulk Richardson number is -0.21 (weakly unstable). To set the bulk Richardson number of a numerical simulation to be similar to that of the wind-tunnel experiment, we apply strong ambient streamwise horizontal velocity with a $5^{\circ} \mathrm{C}$ temperature difference between the bottom surface and the ambient air. The vertical profile of the normalized streamwise horizontal velocity in the numerical simulation is in good agreement with that in the wind-tunnel experiment, although there are some discrepancies in magnitude. The vertical profiles of the normalized temperature are nearly identical to each other. These comparison results support the validity of the temperature wall function implemented in PALM.

Second, the scalar dispersion simulated using PALM is validated against the wind-tunnel data of Meroney et al. (1996). Data for the urban-roughness case, in which the model buildings are aligned in the streamwise direction, are compared with the results of numerical simulation. As in the wind-tunnel experiment, a line source of passive scalar is located at the middle of the street bottom in the numerical simulation. Figure 3 shows normalized scalar concentrations in the numerical simulation

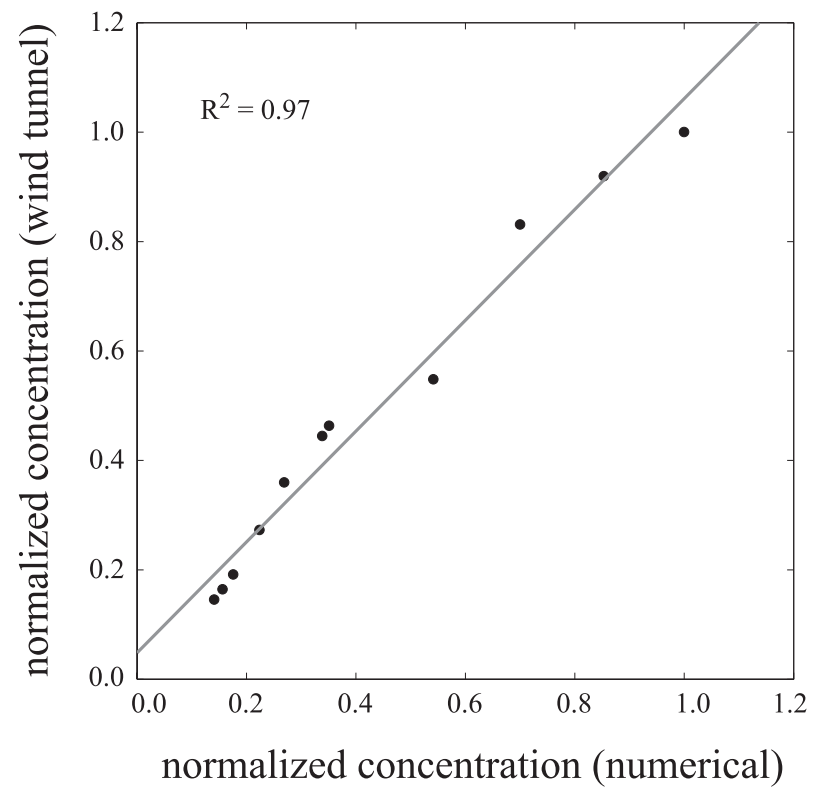

FIG. 3. Normalized scalar concentrations in the numerical simulation and wind-tunnel experiment (Meroney et al. 1996) at 11 locations. Scalar concentrations are normalized by the largest value among the values at the 11 locations. The 11 locations are described in Meroney et al. (1996) and Baik and Kim (2002).

and in the wind-tunnel experiment. Concentrations at 11 locations are plotted, and the values are normalized by the largest value of concentration among the values at the 11 locations. The normalized scalar concentrations in the numerical simulation and in the wind-tunnel experiment are similar to each other.

\section{Results and discussion}

\section{a. Mean flow}

Figure 4 shows streamline fields obtained using timeand spanwise-averaged velocity components in the $\mathrm{NH}$, $\mathrm{UH}, \mathrm{BH}$, and $\mathrm{DH}$ cases. In the $\mathrm{NH}$ case, a primary vortex and small corner vortices appear in the street canyon (Fig. 4a). In the UH case, updraft near the heated upwind building wall strengthens the primary vortex and the primary vortex expands above the rooftop height (Fig. $4 \mathrm{~b})$. The street-bottom heating also reinforces the primary vortex, but the streamline pattern is similar to that of the NH case without any noticeable expansion above the rooftop height. When compared with the $\mathrm{UH}$ and $\mathrm{BH}$ cases, the case with downwind building-wall heating results in considerably different mean flow patterns (Fig. 4d). In the DH case, the primary vortex shrinks and a winding flow appears between the primary vortex and the downwind building wall. Because of the downwind building-wall heating, strong updrafts are generated 
(a) $\mathrm{NH}$

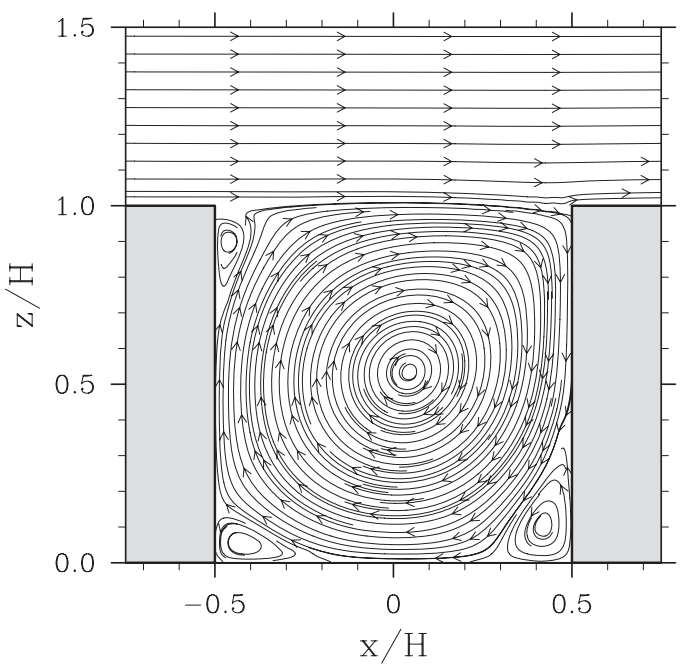

(c) $\mathrm{BH}$

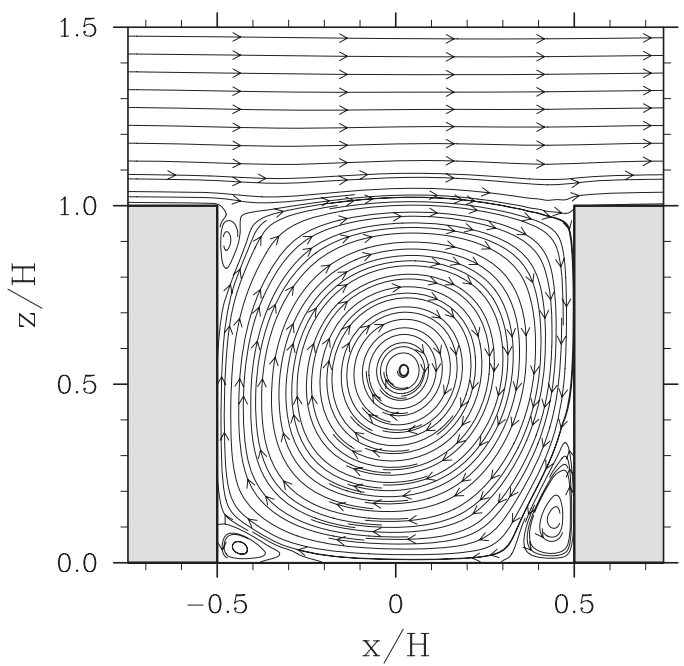

(b) $\mathrm{UH}$

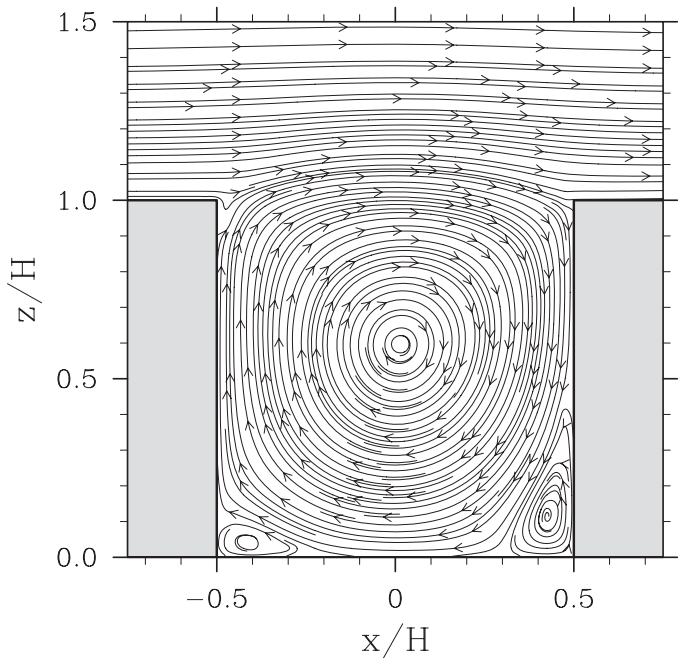

(d) $\mathrm{DH}$

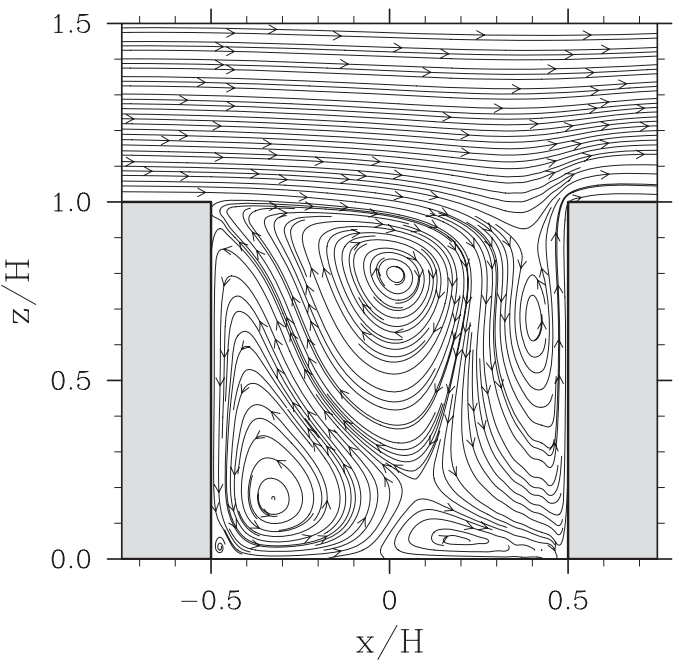

FIG. 4. Streamline fields obtained using time- and spanwise-averaged velocity components in the (a) NH, (b) UH, (c) $\mathrm{BH}$, and (d) DH cases.

along the downwind building wall, and these updrafts block incoming ambient flow. As a result, the ambient air descends not along the downwind building wall but across the region between $x / H=0.1$ and $x / H=0.4$ at the rooftop height. The incoming flow and thermally induced updrafts near the downwind building wall form the winding flow. With the winding flow, the streamlines in the street canyon are no longer completely closed. A similar flow pattern is also observed in the wind-tunnel experiment of Kovar-Panskus et al. (2002). This winding flow is a unique flow pattern that can appear when the mechanical and thermal forcings act together but in opposite ways in a narrow region, and its spatial scale is sensitive to the ambient wind speed and heating intensity.
Thermal effects on the strength of circulation in the street canyon are investigated by analyzing the profiles of time- and spanwise-averaged velocities. Figure 5 shows the vertical profiles of normalized streamwise horizontal velocity at $x / H=0$ and the horizontal profiles of vertical velocity at $z / H=0.5$. In the $\mathrm{UH}$ and $\mathrm{BH}$ cases, the magnitude of normalized streamwise horizontal velocity near the bottom and rooftop height is larger than that of the other cases ( $\mathrm{NH}$ and $\mathrm{DH}$ cases). When the upwind building wall is heated, the flow between $z / H=1.1$ and 1.7 is especially strong because of the buoyant mixing there (Fig. 5a). The horizontal profiles of vertical velocity also confirm the intensified circulation resulting from the upwind building-wall or 
(a)

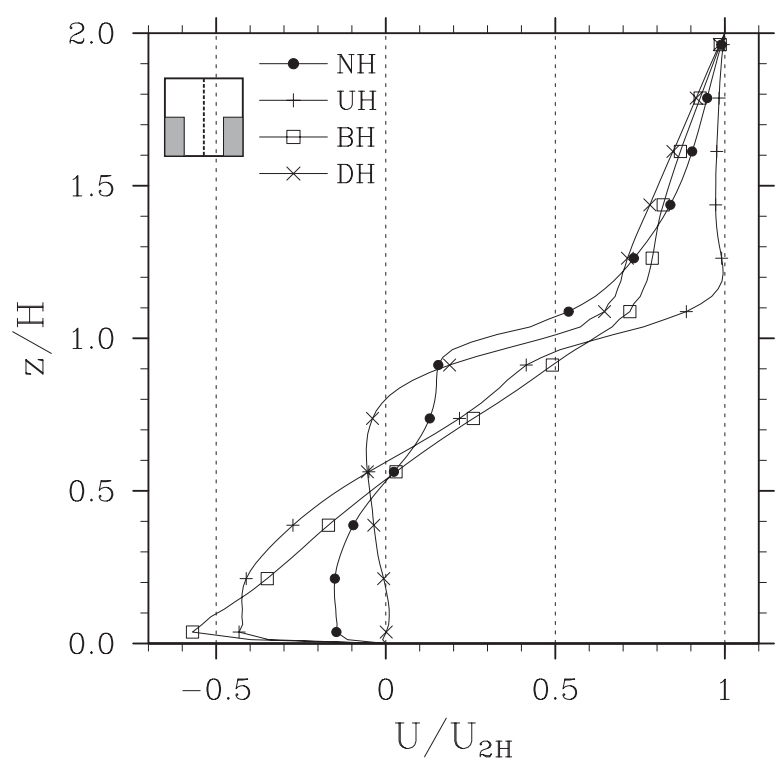

(b)

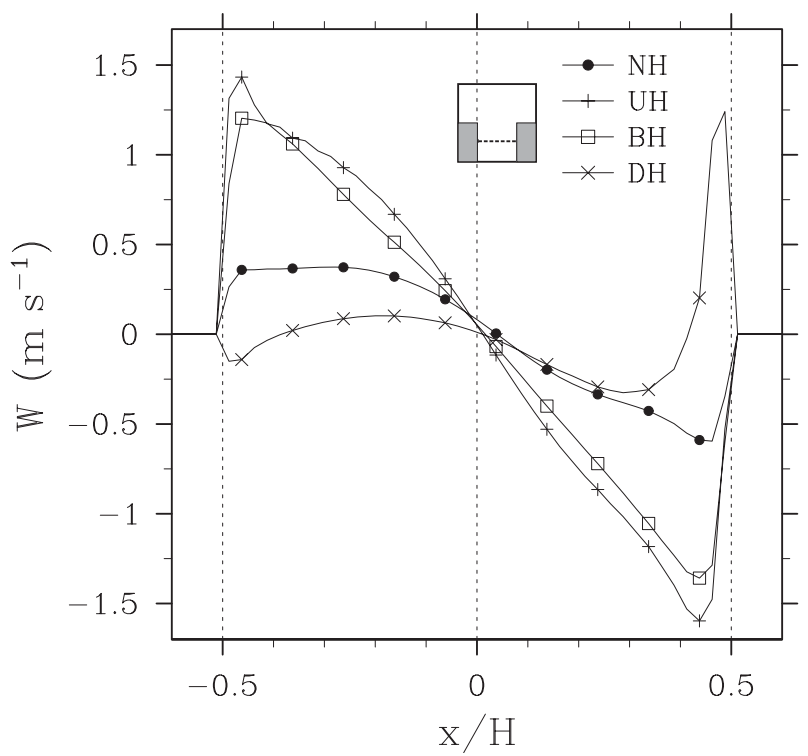

FIG. 5. (a) Vertical profiles of time- and spanwise-averaged normalized streamwise horizontal velocity at $x / H=0$ and (b) horizontal profiles of time- and spanwise-averaged vertical velocity at $z / H=0.5$.

street-bottom heating (Fig. 5b). These results indicate that the upwind building-wall or street-bottom heating strengthens the mechanically induced circulation. By contrast, the mean circulation becomes weaker when the downwind building wall is heated because strong updrafts near the heated downwind building wall oppose the incoming ambient air along the downwind building wall (Fig. 4d). When compared with the NH case, the heating induces stronger updrafts near the upwind building wall in the $\mathrm{UH}$ and $\mathrm{BH}$ cases and updrafts near the downwind building wall in the DH case.

\section{b. Turbulent kinetic energy and turbulent fluxes}

Figure 6 shows the vertical profiles of spanwiseaveraged TKE, streamwise horizontal velocity variance $\overline{u^{\prime 2}}$, and vertical velocity variance $\overline{w^{\prime 2}}$ at $x / H=0$. The variances are normalized by 2 times the value of TKE. Here, $u^{\prime}$ and $w^{\prime}$ denote deviations from the time- and spanwise-averaged velocity components $\bar{U}$ and $\bar{W}$, respectively. The maximum TKE of each case appears at the rooftop height in all cases except the $\mathrm{BH}$ case. The maximum TKE at the rooftop height is attributed to the activity of turbulent eddies starting from the upwind building top and passing above the street canyon. The turbulent eddies are mainly generated by shear instability (see Letzel et al. 2008), and the shear production is the main source of TKE, not only in the $\mathrm{NH}$ case but also in the cases in which the building wall is heated (UH and $\mathrm{DH})$. In the $\mathrm{BH}$ case, the maximum TKE appears at onehalf of the rooftop height, indicating high turbulence activity inside the street canyon. The street-bottom heating makes street-canyon flow highly turbulent, whereas the heating of the upwind or downwind building wall induces turbulent eddies that are locally concentrated near the heated building wall. In fact, snapshots of the instantaneous flow field demonstrate that the streetcanyon flow is more intermittent and fluctuating in the $\mathrm{BH}$ case than in the $\mathrm{UH}$ and $\mathrm{DH}$ cases (not shown). The change of the heating position also modifies the variances of streamwise horizontal velocity and vertical velocity, leading to a modified turbulence structure. In the $\mathrm{NH}$ case, TKE at the rooftop height is mainly composed of the variance of streamwise horizontal velocity. In contrast to the $\mathrm{NH}$ case, heating increases the ratio of vertical velocity variance to TKE at the rooftop height (Fig. 6c), resulting in stronger turbulent fluxes in the vertical direction (see Fig. 7). It is analyzed that the mixing-layer analogy (Raupach et al. 1996) can be applied for the variance of streamwise horizontal velocity (normalized by the friction velocity) at the rooftop height in all cases, including the heating cases, but that the variances of spanwise horizontal velocity and vertical velocity do not satisfy the mixing-layer analogy, even in the neutral case (not shown). Above $z / H=1.4$, the magnitude of the streamwise horizontal velocity variance is larger than that of the vertical velocity variance in all cases. This is a typical characteristic of turbulence in the roughness sublayer influenced by outer-layer disturbances and as observed in the field observation results of Inagaki and Kanda (2008). 
(a)

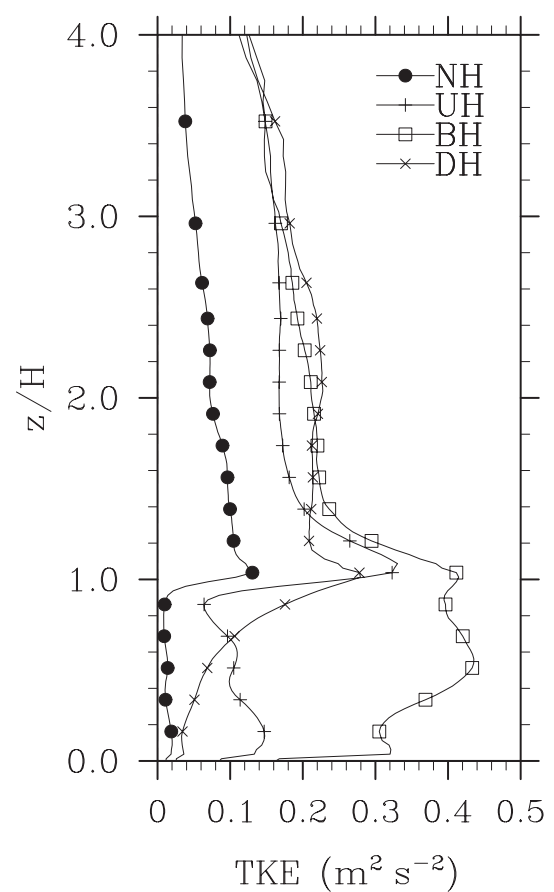

(b)

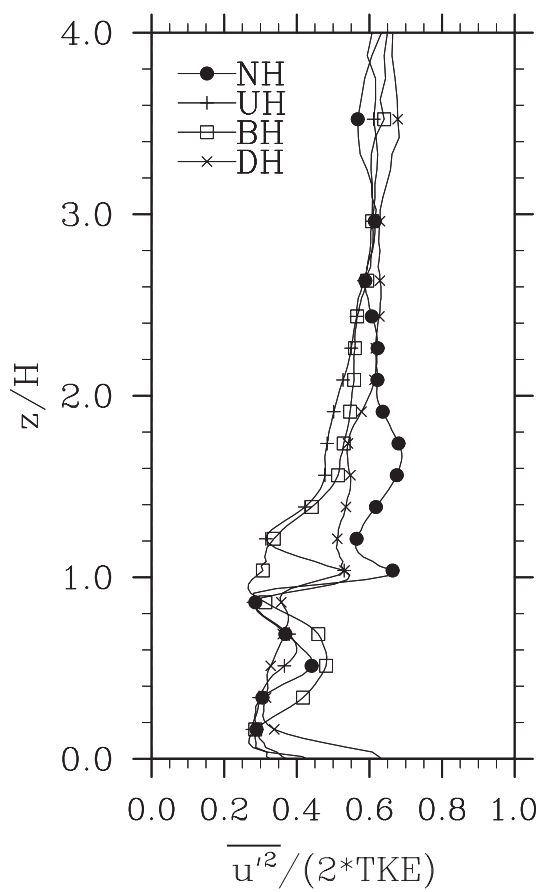

(c)

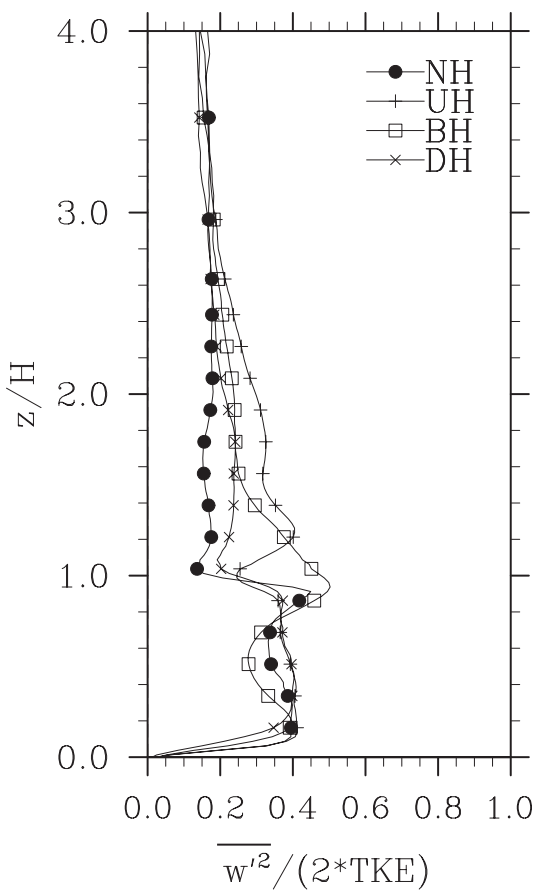

FIG. 6. Vertical profiles of spanwise-averaged (a) TKE, (b) streamwise horizontal velocity variance, and (c) vertical velocity variance at $x / H=0$. The variances are normalized by 2 times the value of TKE.

Figure 7 shows the vertical profiles of the spanwiseaveraged turbulent fluxes of momentum $\overline{u^{\prime} w^{\prime}}$, scalar $\overline{c^{\prime} w^{\prime}}$, and heat $\overline{\theta^{\prime} w^{\prime}}$ at $x / H=0$. Here, $c^{\prime}$ and $\theta^{\prime}$ denote deviations from the time- and spanwise-averaged passive scalar concentration $\bar{C}$ and potential temperature $\bar{\Theta}$, respectively. The turbulent fluxes include only resolved turbulent fluxes $\left(\overline{x^{\prime} w^{\prime}}\right)$ (here, the variable $x$ can be $u, c$, or $\theta$ ) because the magnitude of SGS flux is much smaller than that of resolved-scale flux. In the NH, UH, and $\mathrm{BH}$ cases, all of the vertical profiles have their maximum magnitudes at or near the rooftop height. The vertical profiles of turbulent scalar flux in the $\mathrm{NH}, \mathrm{UH}$, and $\mathrm{BH}$ cases have secondary maximum magnitudes near the street bottom because of the fluctuations of scalar concentration around the scalar source. In the DH case, the turbulent scalar flux has a maximum value at $z / H=0.2$ and not at the rooftop height because wind shear at the rooftop height is weak as a result of the modified flow pattern. The modified flow pattern in the DH case also affects turbulent heat flux in the street canyon. The modified flow pattern induces turbulent eddies concentrated near the downwind building wall, leading to greater heat exchange there. The modified flow pattern decreases the magnitude of turbulent heat flux at $x / H=0$ in the $\mathrm{DH}$ case when compared with the magnitude in other cases ( $\mathrm{UH}$ and $\mathrm{BH}$ cases). The secondary maximum magnitude of $\overline{\theta^{\prime} w^{\prime}}$ near the bottom in the $\mathrm{BH}$ case is due to the bottom heating.

Time- and area-averaged $(-0.5 \leq x / H \leq 0.5 ;-1 \leq y /$ $H \leq 1)$ mean momentum flux $\bar{U} \bar{W}$, turbulent momentum flux $\overline{u^{\prime} w^{\prime}}$, mean scalar flux $\bar{C} \bar{W}$, and turbulent scalar flux $\overline{c^{\prime} w^{\prime}}$ at the rooftop height in the $\mathrm{NH}, \mathrm{UH}, \mathrm{BH}$, and DH cases are shown in Fig. 8. In all of the cases, the mean and turbulent momentum fluxes at the rooftop height are negative (downward) and the magnitude of turbulent momentum flux is much larger than that of mean momentum flux. The magnitude of turbulent momentum flux is much larger in the $\mathrm{UH}, \mathrm{BH}$, and $\mathrm{DH}$ cases than in the $\mathrm{NH}$ case. The enhancement of turbulent momentum flux due to the street-bottom or building-wall heating can be explained by stronger turbulence activity at the rooftop height caused by the intensified in-canyon circulation ( $\mathrm{UH}$ and $\mathrm{BH}$ cases) and the winding flow (DH case). As already mentioned, both the intensified incanyon circulation and the winding flow produce strong fluctuations of vertical velocity at the rooftop height, and therefore the turbulent momentum flux dominates over the mean momentum flux. In all of the cases, the mean and turbulent scalar fluxes at the rooftop height are positive (upward) except the mean scalar flux in the NH case, and the magnitude of turbulent scalar flux is larger than that of mean scalar flux. Note that the magnitude of 
(a)

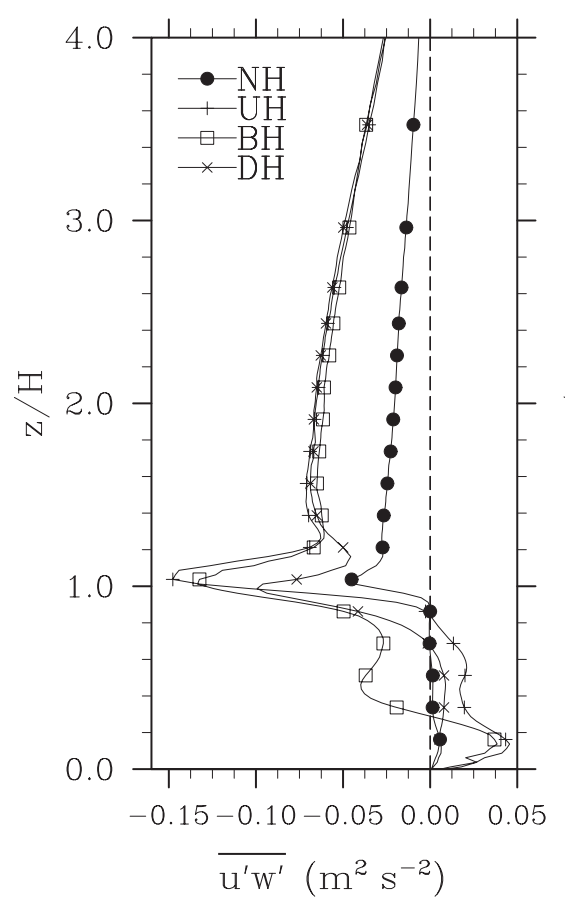

(b)

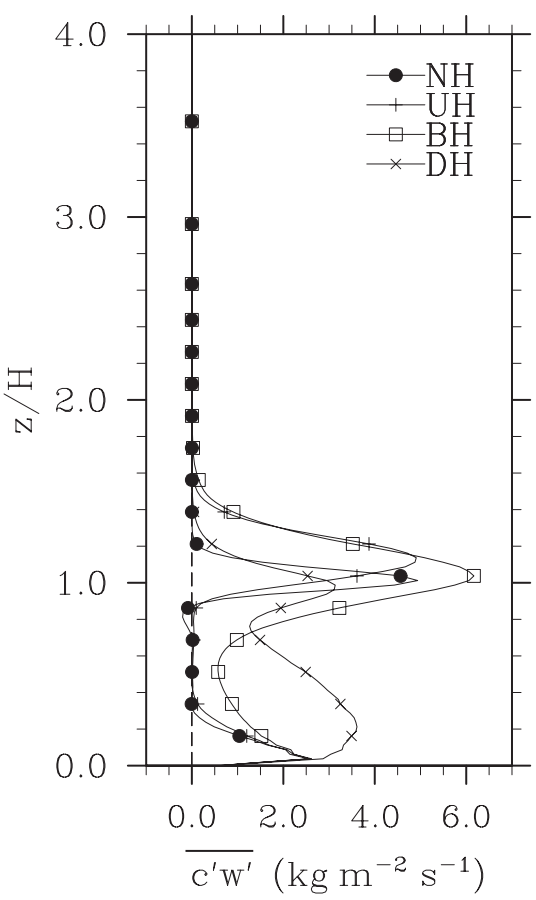

(c)

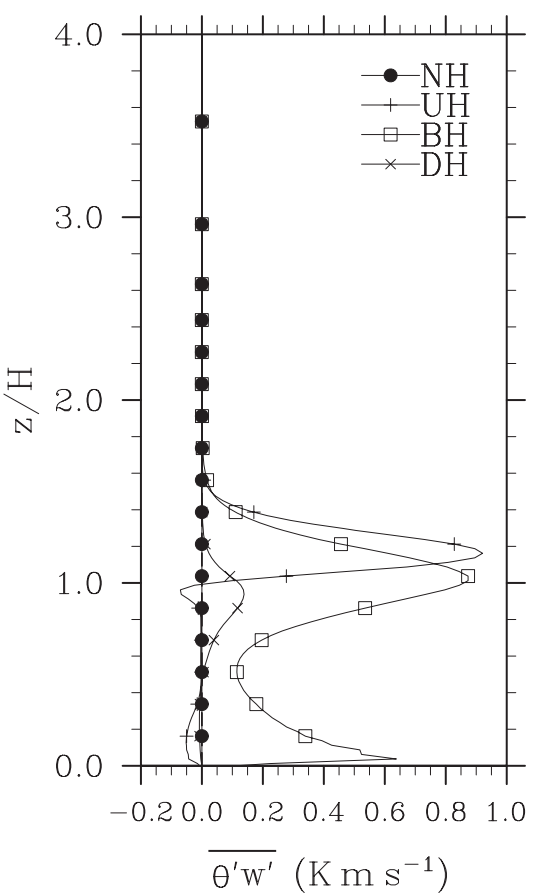

FIG. 7. Vertical profiles of spanwise-averaged (a) turbulent momentum flux, (b) turbulent scalar flux, and (c) turbulent heat flux at $x / H=0$.

turbulent momentum flux (and also turbulent scalar flux) is largest in the $\mathrm{BH}$ case. The magnitude of mean momentum flux (and also mean scalar flux) is smallest in the $\mathrm{BH}$ case, however, although the difference between the $\mathrm{NH}$ and $\mathrm{BH}$ cases is small.

The area-averaged mean momentum flux is actually the sum of dispersive momentum flux and the product of time- and area-averaged streamwise horizontal velocity and vertical velocity. Below the rooftop height, the magnitude of the product is negligible in comparison with that of the dispersive momentum flux. While the dispersive momentum flux is negative at the rooftop height the dispersive momentum flux below the rooftop height is positive, and its magnitude is comparable to the magnitude of turbulent momentum flux in all cases except the DH case (not shown). The positive dispersive momentum flux is attributed to the existence of the primary vortex in the street canyon, and this is consistent with the results of Martilli and Santiago (2007).

To examine thermal effects on instantaneous momentum transport events, the spatial-temporal pattern of normalized $\operatorname{sgn}\left(w^{\prime}\right) \times \max \left(0,-u^{\prime} w^{\prime}\right)$ for the last $300 \mathrm{~s}$ (from 10500 to $10800 \mathrm{~s}$ ) at the rooftop height is plotted in Fig. 9. The quantity $\operatorname{sgn}\left(w^{\prime}\right) \times \max \left(0,-u^{\prime} w^{\prime}\right)$ is the product of the sign of vertical velocity perturbation and the magnitude of downward turbulent momentum flux ( 0 for upward turbulent momentum flux). Thus, the spatial-temporal fields of $\operatorname{sgn}\left(w^{\prime}\right) \times \max \left(0,-u^{\prime} w^{\prime}\right)$ clearly visualize the distribution of events of downward momentum transport accompanying updrafts (ejections) and those accompanying downdrafts (sweeps). In the figure, $\operatorname{sgn}\left(w^{\prime}\right) \times \max \left(0,-u^{\prime} w^{\prime}\right)$ is normalized by the magnitude of time- and area-averaged turbulent momentum flux $\left|\overline{u^{\prime} w^{\prime}}\right|$ at the rooftop height to emphasize intermittent and dominant turbulent events. In the $\mathrm{NH}$ case, the perturbation patterns are band shaped with a constant slope, corresponding to a reciprocal of transfer velocity. This indicates that momentum is mostly transported by turbulent eddies that move with a constant velocity (approximately $0.7 \mathrm{~m} \mathrm{~s}^{-1}$ ). The slope of perturbations in the $\mathrm{UH}$ and $\mathrm{BH}$ cases is gentler than that in the $\mathrm{NH}$ case, corresponding to a faster transfer velocity (approximately $1.5 \mathrm{~m} \mathrm{~s}^{-1}$ ) at the rooftop height. The estimated transfer velocity is nearly identical to the mean streamwise horizontal velocity at the rooftop height in all cases. Strong turbulent events occur more frequently in the UH case because of the strong flow at the rooftop height. Although the band-shaped patterns are still maintained in the UH case, the magnitude of momentum transport by individual turbulent events increases, especially near the upwind building wall. Contrary to the UH case, the street-bottom heating induces more intermittent turbulent eddies, and the eddies sometimes have bandshaped and sometimes have lump-shaped patterns in the 
(a)

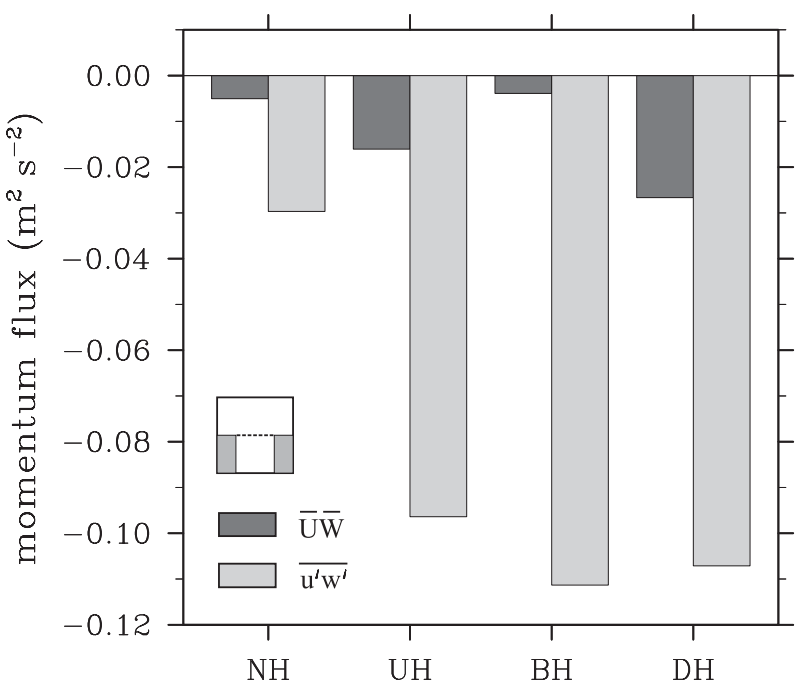

(b)

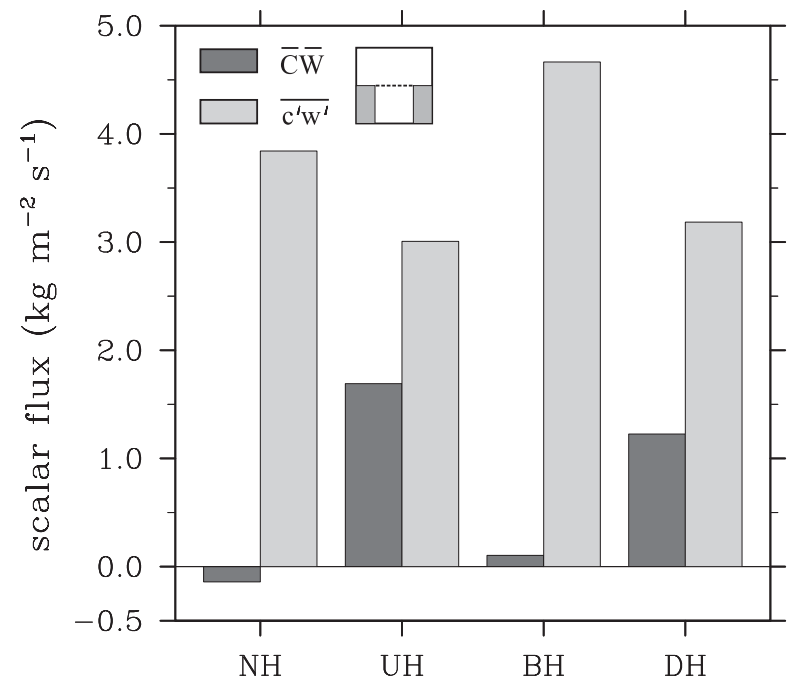

FIG. 8. Time- and area-averaged $(-0.5 \leq x / H \leq 0.5 ;-1 \leq y / H \leq$ 1) (a) mean and turbulent momentum fluxes and (b) mean and turbulent scalar fluxes at the rooftop height in the $\mathrm{NH}, \mathrm{UH}, \mathrm{BH}$, and $\mathrm{DH}$ cases.

$x-t$ plane. In the $\mathrm{BH}$ case, heating is not localized but spreads throughout the entire street canyon, and this kind of heating induces more fluctuating flow therein and more intermittent turbulence signals at the rooftop height. The downwind building-wall heating induces strong updrafts near the downwind building wall, and turbulent eddies related to the updrafts appear as lumpshaped perturbations in the $x-t$ plane. Lump-shaped perturbations coexist with weaker band-shaped perturbations (Fig. 9d), and turbulent eddies corresponding to the two kinds of perturbations dominate mixing at the rooftop height.
Figure 10 shows the spatial-temporal pattern of normalized $\operatorname{sgn}\left(w^{\prime}\right) \times \max \left(0, c^{\prime} w^{\prime}\right)$ for the last $300 \mathrm{~s}$ at the rooftop height. Also in this figure, $\operatorname{sgn}\left(w^{\prime}\right) \times \max \left(0, c^{\prime} w^{\prime}\right)$ is normalized by the magnitude of time- and areaaveraged turbulent scalar flux $\left|\overline{c^{\prime} w^{\prime}}\right|$ at the rooftop height. As in momentum transport events, band-shaped scalar transport events appear in the $\mathrm{NH}$ case. This similarity indicates that turbulent eddies generated by shear instability dominate passive scalar transport at the rooftop height. At the rooftop height, sweep events draw ambient fresh air into the street canyon and ejection events get passive scalar out from the street canyon, resulting in a net upward scalar transport. In the UH case, both shear instability and heating generate turbulent eddies near the upwind building wall. Scalarescaping events and above-canyon-air-incoming events are concentrated in the upwind region between $x / H=$ -0.5 and $x / H=0$. In the BH case, distinctly intermittent transport patterns are repeated in the instantaneous turbulent scalar flux field. In the DH case, lump-shaped scalar transport events dominate scalar transport at the rooftop height, especially near the downwind building wall. Near the heated downwind building wall, the scalar transport events induced by local downdrafts are lump shaped. When compared with the momentum transport events, thermal effects due to the change of the heating position are more distinct in the scalar transport events at the rooftop height because scalar transport events are more dependent on the fluctuations of vertical velocity and the intensity of updrafts. Overall, the timing of the momentum (scalar) transport seems to be determined by outer flow in the $\mathrm{NH}$ and $\mathrm{UH}$ cases where sweep events are dominant, whereas the timing seems to be dependent on both outer flow and in-canyon flow in the $\mathrm{BH}$ and $\mathrm{DH}$ cases where ejection events are comparable to sweep events.

\section{c. Conditional statistics of turbulent momentum flux}

To investigate thermal effects on the turbulence structure in the shear layer, a quadrant analysis is performed. Quadrant analyses have been used in numerous studies, including plant-canopy turbulence (Shaw et al. 1983) and urban surface-layer turbulence (Raupach 1981; Rotach 1993; Cui et al. 2004; Christen et al. 2007). The instantaneous turbulent momentum flux $u^{\prime} w^{\prime}$ (or instantaneous Reynolds stress) at one position can be classified into four quadrants: outward interaction $\left(u^{\prime}>0 ; w^{\prime}>0\right)$, ejection $\left(u^{\prime}<0 ; w^{\prime}>0\right)$, inward interaction $\left(u^{\prime}<0 ; w^{\prime}<\right.$ $0)$, and sweep $\left(u^{\prime}>0 ; w^{\prime}<0\right)$. Following the method of Raupach (1981), the frequency (or time fraction) and the contribution to turbulent momentum flux of quadrant $i$ are expressed by 
(a)

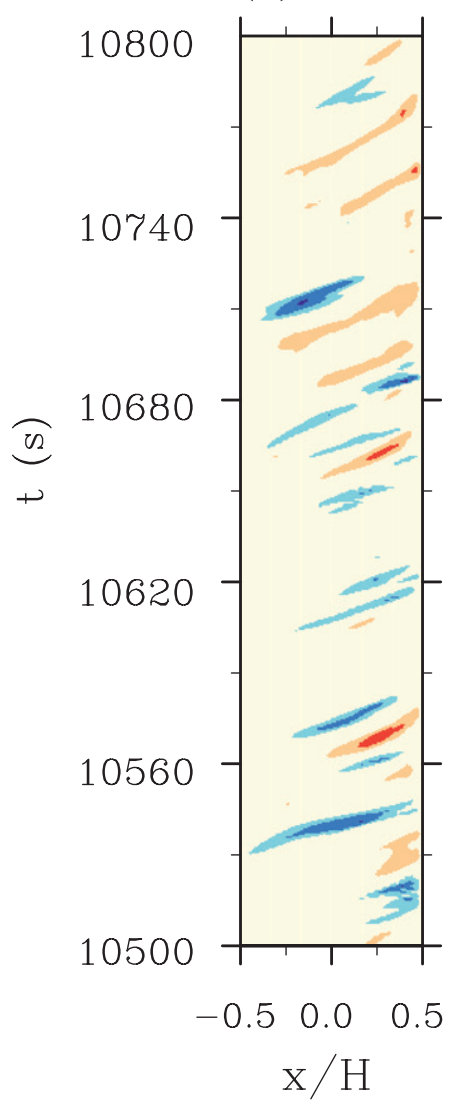

(b)

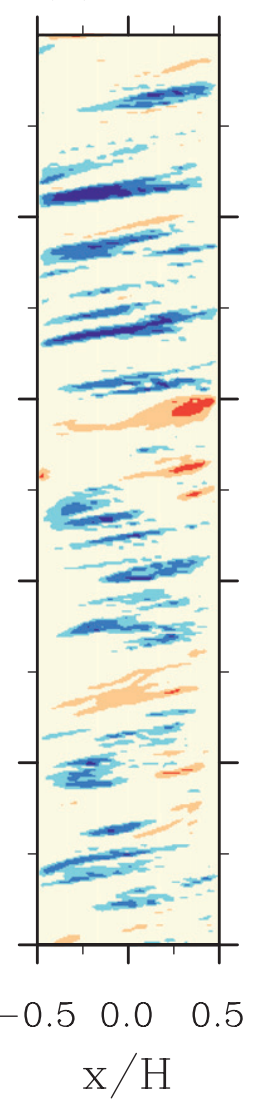

(c)

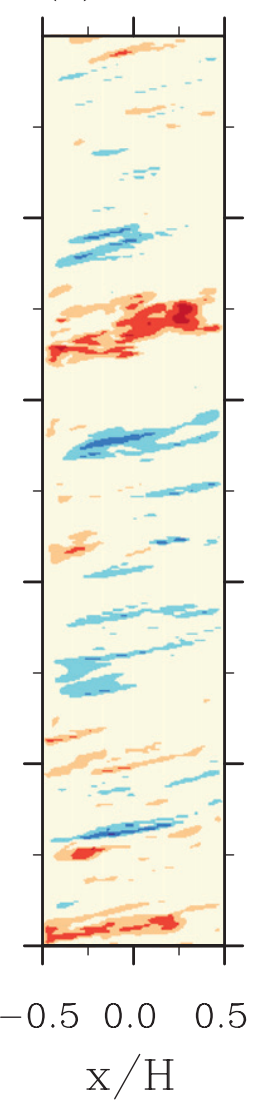

(d)

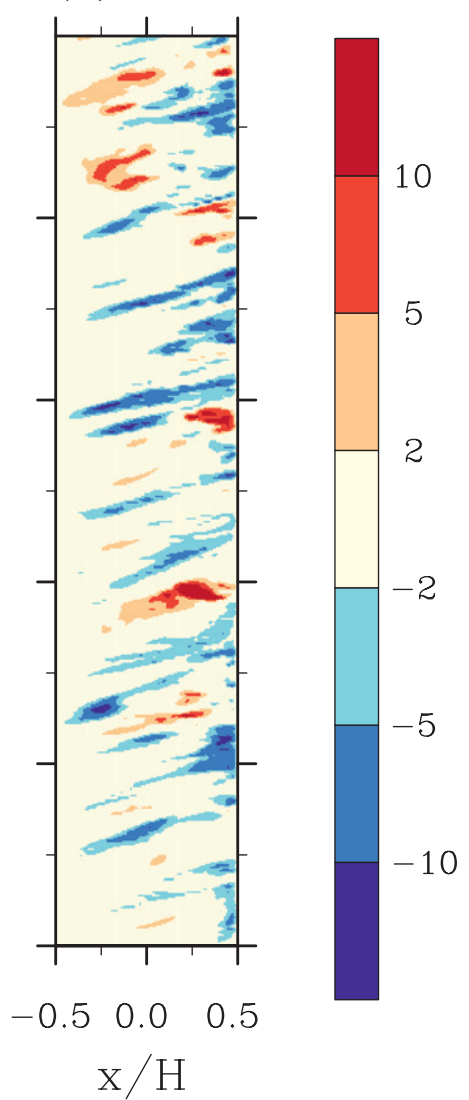

FIG. 9. Spatial-temporal pattern of normalized $\operatorname{sgn}\left(w^{\prime}\right) \times \max \left(0,-u^{\prime} w^{\prime}\right)$ at the rooftop height in the (a) $\mathrm{NH}$, (b) UH, (c) BH, and (d) DH cases. The instantaneous downward momentum flux is normalized by the magnitude of time- and area-averaged turbulent momentum flux $\left|\overline{u^{\prime} w^{\prime}}\right|$ at the rooftop height.

$$
\begin{gathered}
T_{i}=T^{-1} \int_{T} I_{i}\left[u^{\prime}(t), w^{\prime}(t)\right] d t \quad \text { and } \\
\left\langle u^{\prime} w^{\prime}\right\rangle_{i}=T^{-1} \int_{T} u^{\prime}(t) w^{\prime}(t) I_{i}\left[u^{\prime}(t), w^{\prime}(t)\right] d t,
\end{gathered}
$$

where the indicator function $I_{i}$ is 1 if $\left(u^{\prime}, w^{\prime}\right)$ is in quadrant $i$ and is 0 if otherwise. The stress fraction, a ratio of the contribution of quadrant $i$ to turbulent momentum flux (Reynolds stress), is

$$
S_{i}=\left\langle u^{\prime} w^{\prime}\right\rangle_{i} / \overline{u^{\prime} w^{\prime}}
$$

Figure 11 shows the frequency $T_{i}$ and the stress fraction $S_{i}$ of turbulent events at $(x, y, z)=(0,0, H)$ and $(0,0,2.5 H)$. In this study, the results at one point (not spanwise averaged) are presented because the spanwise variation of conditional statistics is negligible. At the rooftop height, ejection and sweep events occur most frequently and contribute to most of turbulent momentum flux. In the NH and UH cases, sweep events contribute more to turbulent momentum flux than do ejection events, although ejection events occur more frequently. This kind of trend is one of the typical turbulence structures at the top of the canopy layer because of the dominance of turbulent eddies that are generated by shear instability. This result is consistent with the results of Cui et al. (2004). When the street bottom or downwind building wall is heated, the frequency of ejection events is close to that of sweep events and the difference in the stress fraction between the two events becomes smaller than the difference in the $\mathrm{NH}$ and $\mathrm{UH}$ cases. This emphasizes the important role ejection events play in momentum transport in the $\mathrm{BH}$ and $\mathrm{DH}$ cases. In the $\mathrm{BH}$ and $\mathrm{DH}$ cases, buoyancy-generated turbulent eddies appear as strong ejection events at the rooftop height (corresponding to the lump-shaped perturbations in Fig. 9) and change the turbulence structure in the shear layer (including the rooftop height). The frequency and stress fraction in the roughness sublayer 
(a)

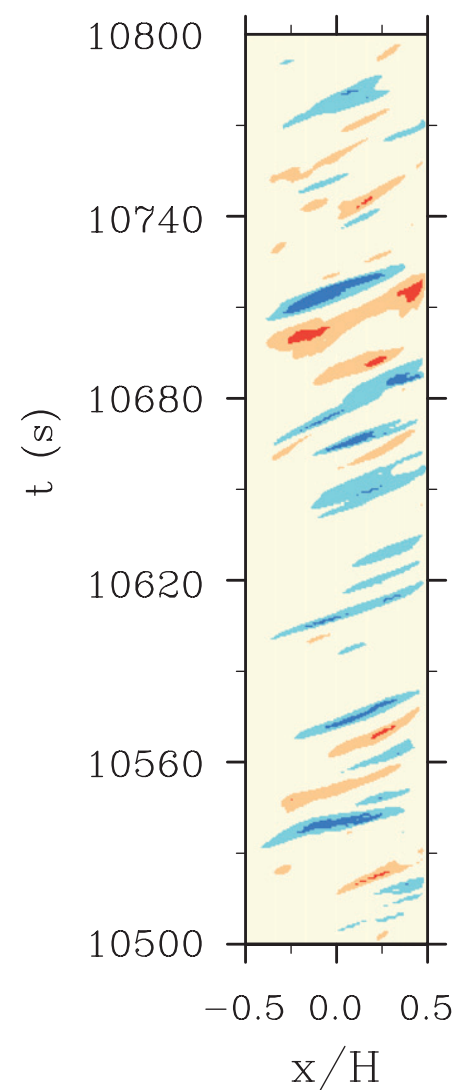

(b)

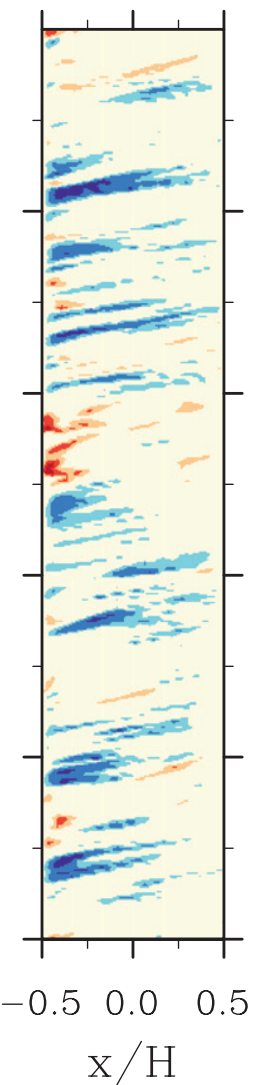

(c)

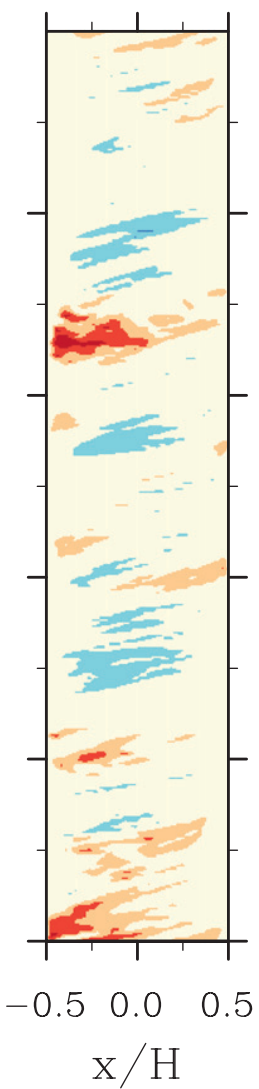

(d)

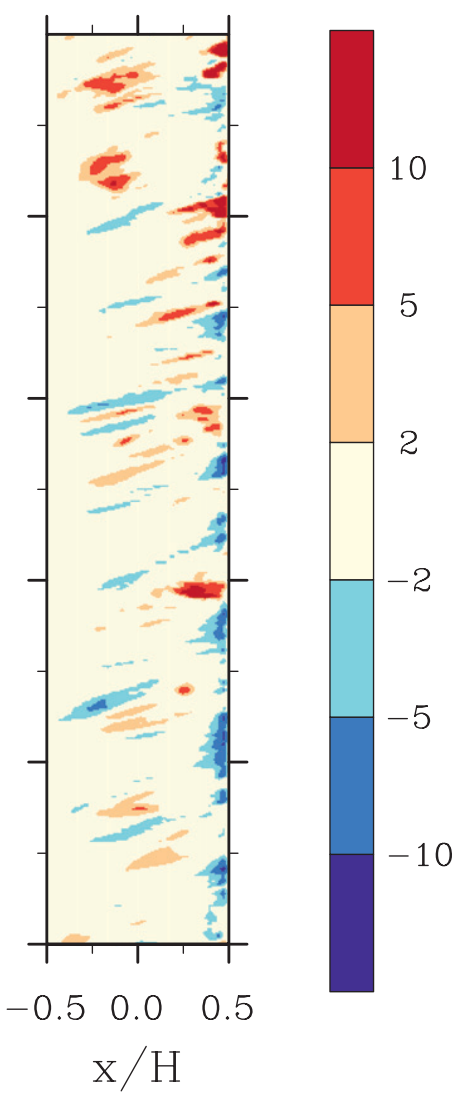

FIG. 10. Spatial-temporal pattern of normalized $\operatorname{sgn}\left(w^{\prime}\right) \times \max \left(0, c^{\prime} w^{\prime}\right)$ at the rooftop height in the (a) NH, (b) UH, (c) BH, and (d) DH cases. The instantaneous upward scalar flux is normalized by the magnitude of time- and area-averaged turbulent scalar flux $\left|\overline{c^{\prime} w^{\prime}}\right|$ at the rooftop height.

$(z / H=2.5)$ are also shown in Fig. 11 to compare the turbulence structure in the roughness sublayer with that in the shear layer. In the roughness sublayer, ejection events contribute more to turbulent momentum flux than do sweep events in the $\mathrm{NH}$ and $\mathrm{DH}$ cases and vice versa in the $\mathrm{UH}$ and $\mathrm{BH}$ cases. The dominance of sweep events over ejection events in the $\mathrm{NH}$ and $\mathrm{UH}$ cases in the shear layer does not appear any more in the roughness sublayer. In addition, the difference in stress fraction has less influence on turbulent momentum flux in the roughness sublayer than in the shear layer because the magnitude of turbulent momentum flux in the roughness sublayer is much smaller than that at the rooftop height. Thus, the only common characteristic of turbulence structures at both heights is the dominance of ejection and sweep events over events with outward and inward interaction in turbulent momentum flux. This indicates that the effects of differential heating positions are confined to the street canyon and the shear layer just above the rooftop height.

\section{Summary and conclusions}

Using an LES model that includes a temperature wall function, thermal effects on turbulent flow and dispersion in and above a street canyon with a street aspect ratio of 1 were numerically investigated. Four numerical simulations were performed: cases with no heating (neutral), upwind building-wall heating, street-bottom heating, and downwind building-wall heating. A stronger primary vortex in the street canyon appears in the cases with upwind building-wall heating and street-bottom heating than in the neutral case, and the downwind building-wall heating produces an interesting in-canyon flow pattern with a shrunken primary vortex and a winding flow. The differential heating produces higher TKE and stronger turbulent fluxes at the rooftop height. In the differential-heating cases, buoyancy-generated turbulent eddies as well as shear-generated turbulent eddies contribute to the turbulent transport of momentum and scalar and band-shaped or lump-shaped 
(a)

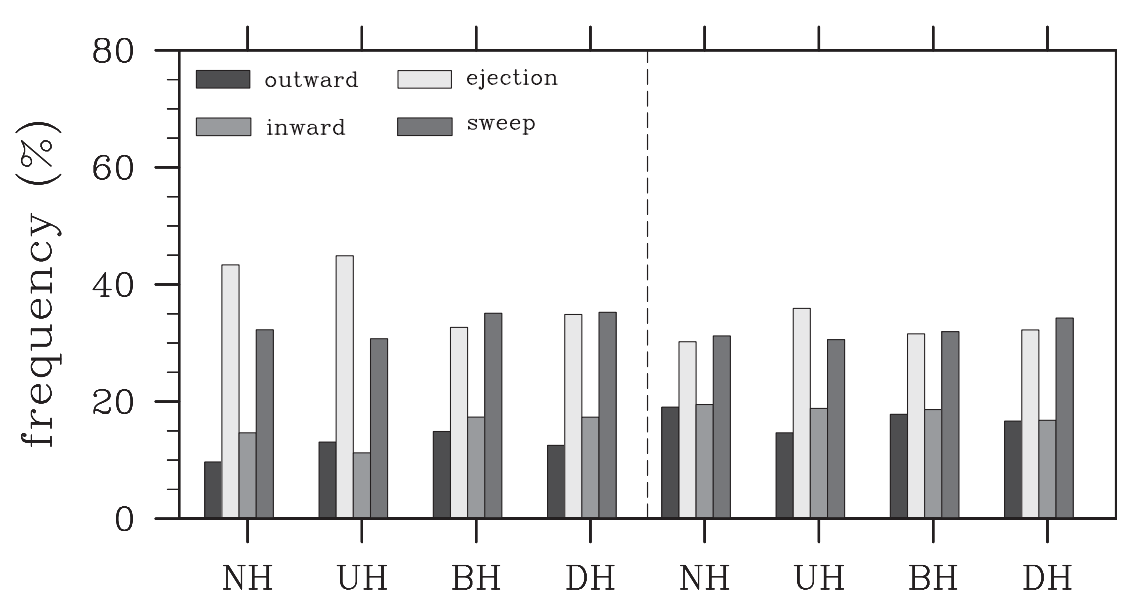

(b)

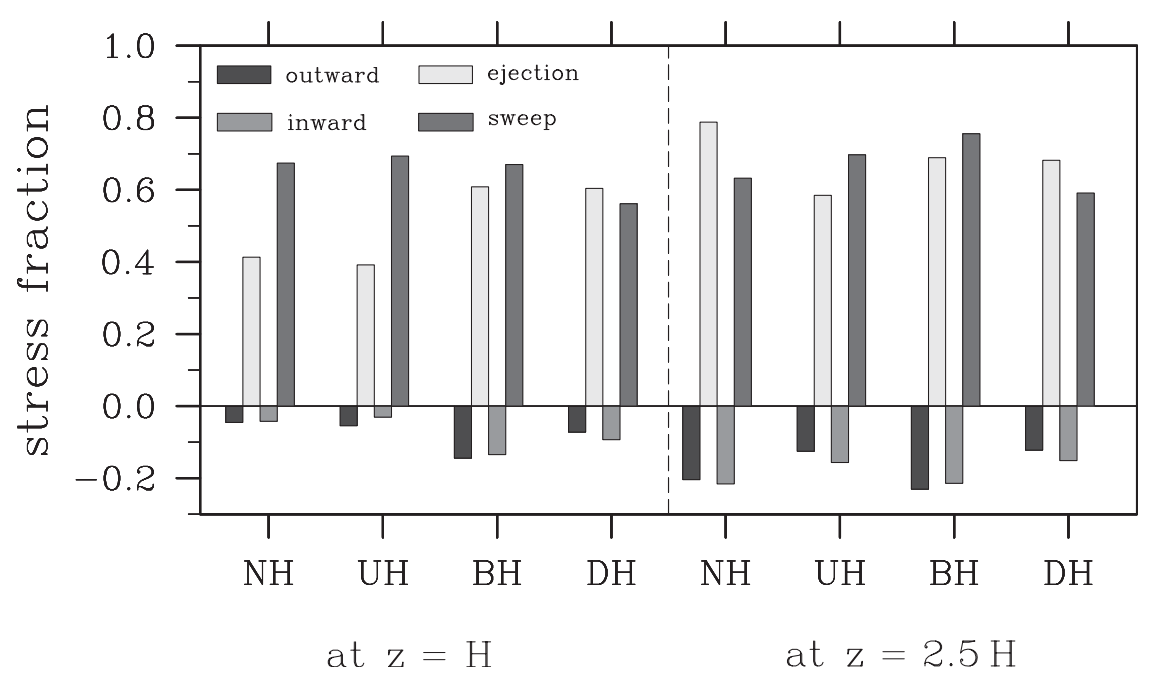

FIG. 11. (a) Frequency $T_{i}$ and (b) stress fraction $S_{i}$ of the four quadrant events at $(x, y, z)=$ $(0,0, H)$ and $(0,0,2.5 H)$.

perturbation patterns appear at the rooftop height. At the rooftop height, in the neutral case and in the case with upwind building-wall heating, sweep events are less frequent but contribute more to turbulent momentum flux than do ejection events. In the cases with streetbottom heating and downwind building-wall heating, ejection and sweep events are similar in occurrence and their contributions to turbulent momentum flux are comparable. It was found that these differences are not distinct at a height well above the rooftop.

In this study, the statistical characteristics of turbulence structure in and above a street canyon affected by street-bottom or building-wall heating were examined, but the relationship between individual turbulent eddies and their associated scalar dispersion was not fully explained. Understanding instantaneous interactions between individual turbulent eddies and scalar dispersion is one of the important problems in the field of urban flow and dispersion. This research would be undertaken using methods such as conditional averaging and proper orthogonal decomposition (Coceal et al. 2007; Huang et al. 2009). In this study, only one street aspect ratio and one heating intensity were considered. It is well known that the street aspect ratio and heating intensity greatly influence flow and dispersion in street canyons. Further study is needed to examine changes in turbulence structure with different aspect ratios and heating intensities.

Last, it is noted that this study focused on turbulence structures in and above a street canyon, influenced by the local heating. In addition to the local heating, large-scale turbulent motions appearing above building 
canopies also affect turbulence structures below them in a way similar to the top-down mechanism (Hunt and Morrison 2000). Although turbulent eddies above the building canopy are simulated in this study, the size of the simulated eddies is limited to the horizontal domain size $(2 H)$. Further study with a larger horizontal domain that can cover large-scale turbulent motions is needed to better understand turbulence structures in and above street canyons.

Acknowledgments. The authors are grateful to two anonymous reviewers for providing valuable comments on this work. This work was supported by a National Research Foundation of Korea (NRF) grant funded by the South Korean government (MEST) (2011-0017041) and by the Brain Korea 21 Project (through the School of Earth and Environmental Sciences, Seoul National University). This work was also supported by the German Research Foundation under Grant RA 617/15-2.

\section{REFERENCES}

Baik, J.-J., and J.-J. Kim, 2002: On the escape of pollutants from urban street canyons. Atmos. Environ., 36, 527-536.

Christen, A., E. V. Gorsel, and R. Vogt, 2007: Coherent structures in urban roughness sublayer turbulence. Int. J. Climatol., 27, 1955-1968.

Coceal, O., A. Dobre, T. G. Thomas, and S. E. Belcher, 2007: Structure of turbulent flow over regular arrays of cubical roughness. J. Fluid Mech., 589, 375-409.

Cui, Z., X. M. Cai, and C. J. Baker, 2004: Large-eddy simulation of turbulent flow in a street canyon. Quart. J. Roy. Meteor. Soc., 130, 1373-1394.

Deardorff, J. W., 1980: Stratocumulus-capped mixed layers derived from a three-dimensional model. Bound.-Layer Meteor., 18, 495-527.

Huang, J., M. Cassiani, and J. D. Albertson, 2009: Analysis of coherent structures within the atmospheric boundary layer. Bound.-Layer Meteor., 131, 147-171.

Hunt, J. C. R., and J. F. Morrison, 2000: Eddy structure in turbulent boundary layers. Eur. J. Mech.-B/Fluids, 19, 673-694.

Inagaki, A., and M. Kanda, 2008: Turbulent flow similarity over an array of cubes in near-neutrally stratified atmospheric flow. J. Fluid Mech., 615, 101-120.

— and - 2010: Organized structure of active turbulence over an array of cubes within the logarithmic layer of atmospheric flow. Bound.-Layer Meteor., 135, 209-228.

Kanda, M., R. Moriwaki, and F. Kasamatsu, 2004: Large-eddy simulation of turbulent organized structures within and above explicitly resolved cube arrays. Bound.-Layer Meteor., 112, 343-368.
Kim, J.-J., and J.-J. Baik, 1999: A numerical study of thermal effects on flow and pollutant dispersion in urban street canyons. J. Appl. Meteor., 38, 1249-1261.

Kovar-Panskus, A., L. Moulinneuf, E. Savory, A. Abdelqari, J.-F. Sini, J.-M. Rosant, A. Robins, and N. Toy, 2002: A wind tunnel investigation of the influence of solar-induced wall-heating on the flow regime within a simulated urban street canyon. Water Air Soil Pollut.: Focus, 2, 555-571.

Letzel, M. O., M. Krane, and S. Raasch, 2008: High resolution urban large-eddy simulation studies from street canyon to neighbourhood scale. Atmos. Environ., 42, 8770-8784.

Louka, P., G. Vachon, J.-F. Sini, P. G. Mestayer, and J.-M. Rosant, 2002: Thermal effects on the airflow in a street canyonNantes'99 experimental results and model simulations. Water Air Soil Pollut.: Focus, 2, 351-364.

Martilli, A., and J. L. Santiago, 2007: CFD simulation of airflow over a regular array of cubes. Part II: Analysis of spatial average properties. Bound.-Layer Meteor., 122, 635654.

Meroney, R. N., M. Pavageau, S. Rafailidis, and M. Schatzmann, 1996: Study of line source characteristics for 2-D physical modelling of pollutant dispersion in street canyons. J. Wind Eng. Ind. Aerodyn., 62, 37-56.

Offerle, B., I. Eliasson, C. S. B. Grimmond, and B. Holmer, 2007: Surface heating in relation to air temperature, wind and turbulence in an urban street canyon. Bound.-Layer Meteor., 122, 273-292.

Raasch, S., and M. Schröter, 2001: PALM-A large-eddy simulation model performing on massively parallel computers. $\mathrm{Me}$ teor. Z., 10, 363-372.

Raupach, M. R., 1981: Conditional statistics of Reynolds stress in rough-wall and smooth-wall turbulent boundary layers. J. Fluid Mech., 108, 363-382.

_ J. J. J. Finnigan, and Y. Brunet, 1996: Coherent eddies and turbulence in vegetation canopies: The mixing-layer analogy. Bound.-Layer Meteor., 78, 351-382.

Rotach, M. W., 1993: Turbulence close to a rough urban surface, Part I: Reynolds stress. Bound.-Layer Meteor., 65, 1-28.

- 1995: Profiles of turbulence statistics in and above an urban street canyon. Atmos. Environ., 29, 1473-1486.

Shaw, R. H., J. Tavangar, and D. P. Ward, 1983: Structure of the Reynolds stress in a canopy layer. J. Climate Appl. Meteor., 22, 1922-1931.

Shen, S., and M. Y. Leclerc, 1997: Modelling the turbulence structure in the canopy layer. Agric. For. Meteor., 87, 3-25.

Sini, J.-F., S. Anquetin, and P. G. Mestayer, 1996: Pollutant dispersion and thermal effects in urban street canyons. Atmos. Environ., 30, 2659-2677.

Uehara, K., S. Murakami, S. Oikawa, and S. Wakamatsu, 2000: Wind tunnel experiments on how thermal stratification affects flow in and above urban street canyons. Atmos. Environ., 34, 1553-1562.

Xie, X., C.-H. Liu, D. Y. C. Leung, and M. K. H. Leung, 2006: Characteristics of air exchange in a street canyon with ground heating. Atmos. Environ., 40, 6396-6409. 